\title{
Higher order antibunching in intermediate states
}

\author{
Amit Verma $a^{1}$, Navneet K Sharma $2^{2}$ and Anirban Pathak ${ }^{3}$ \\ Department of Physics, JIIT University, A-10, Sectror-62, Noida, UP-201307, India.
}

\begin{abstract}
Since the introduction of binomial state as an intermediate state, different intermediate states have been proposed. Different nonclassical effects have also been reported in these intermediate states. But till now higher order antibunching or higher order subpoissonian photon statistics is predicted only in one type of intermediate state, namely shadowed negative binomial state. Recently we have shown the existence of higher order antibunching in some simple nonlinear optical processes to establish that higher order antibunching is not a rare phenomenon (J. Phys. B 39 (2006) 1137). To establish our earlier claim further, here we have shown that the higher order antibunching can be seen in different intermediate states, such as binomial state, reciprocal binomial state, hypergeometric state, generalized binomial state, negative binomial state and photon added coherent state. We have studied the possibility of observing the higher order subpoissonian photon statistics in different limits of intermediate states. The effect of different control parameters have also been studied in this connection and it has been shown that the depth of nonclassicality can be tuned by controlling various physical parameters.
\end{abstract}

\section{Introduction}

An intermediate state is a quantum state which reduces to two or more distinguishably different states (normally, distinguishable in terms of photon number distribution) in different limits. In 1985, such a state was first time introduced by Stoler et al. [1. To be precise, they introduced Binomial state (BS) as a state which is intermediate between the most nonclassical number state $|n\rangle$ and the most classical coherent state $|\alpha\rangle$. They defined BS as

$$
|p, M\rangle=\sum_{n=0}^{M} \quad B_{n}^{M}|n\rangle=\sum_{n=0}^{M} \sqrt{{ }^{M} C_{n} p^{n}(1-p)^{M-n}}|n\rangle \quad 0 \leq p \leq 1 .
$$

This stat $\epsilon^{4}$ is called intermediate state as it reduces to number state in the limit $p \rightarrow 0$ and $p \rightarrow 1$ (as $|0, M\rangle=|0\rangle$ and $|1, M\rangle=|M\rangle$ ) and in the limit of $M \rightarrow \infty, p \rightarrow 1$, where $\alpha$ is a real constant, it reduces to a coherent state with real amplitude. Since the introduction of BS as an intermediate state, it was always been of interest to quantum optics, nonlinear optics, atomic physics and molecular physics community. Consequently, different properties of binomial states have been studied [2 27]. In these studies it has been observed that the nonclassical phenomena (such as, antibunching, squeezing and higher order squeezing) can be seen in BS. This trend of search for nonclassicality in Binomial state, continued in nineties. In one hand, several versions of generalized BS have been proposed [3-5] and in the other hand, people went beyond binomial states and proposed several other form of intermediate states (such as, excited binomial state 6], odd excited binomial state [7, hypergeometric state [8, negative hypergeometric state [9, reciprocal binomial state [10, shadowed state [11], shadowed negative binomial state [12] and photon added coherent state 13 etc.). The studies in the nineties were mainly limited to theoretical predictions but the recent developments in the experimental techniques made it possible to verify some of those theoretical predictions. For example, we can note that, as early as in 1991 Agarwal and Tara [13 had introduced photon added coherent state as

$$
|\alpha, m\rangle=\frac{a^{\dagger m}|\alpha\rangle}{\left\langle a^{m} a^{\dagger m}\right\rangle}
$$

\footnotetext{
${ }^{1}$ amit.verma@jiit.ac.in

${ }^{2}$ navneetk.sharma@jiit.ac.in

3 anirban.pathak@jiit.ac.in

${ }^{4}$ The state is named as binomial state because the photon number distribution associated with this state $\left(i . e .\left|B_{n}^{M}\right|^{2}\right)$ is simply a binomial distribution.
} 
(where $m$ is an integer and $|\alpha\rangle$ is coherent state) but the experimental generation of the state has happened only in recent past when Zavatta, Viciani and Bellini [14] succeed to produce it in 2004. It is easy to observe that (2) represents an intermediate state, since it reduces to coherent state in the limit $m \rightarrow 0$ and to number state in the limit $\alpha \rightarrow 0$. This state can be viewed as a coherent state in which additional $m$ photon are added. The photon number distribution of all the above mentioned states are different but all these states belong to a common family of states called intermediate state. It has also been found that most of these intermediate states show antibunching, squeezing, higher order squeezing, subpoissonian photon statistics etc. but higher order antibunching has been reported only in shadowed negative binomial state [12. Inspired by these observations, many schemes to generate intermediate states have been proposed in recent past 14,17 .

The reason behind the study of nonclassical properties of intermediate states lies in the fact that the most of the interesting recent developments in quantum optics have arisen through the nonclassical properties of the radiation field only. For example, antibunching and squeezing, which do not have any classical analogue 18,20], have extensively been studied in last thirty years. But the majority of these studies are focused on lowest order nonclassical effects. Higher order extensions of these nonclassical states have only been introduced in recent past 21 24]. Among these higher order nonclassical effects, higher order squeezing has already been studied in detail [21, 22, 25, 26] but the higher order antibunching (HOA) is not yet studied rigourously.

The idea of HOA was introduced by Lee in a pioneering paper 23 in 1990, since then it has been predicted in two photon coherent state [23, shadowed negative binomial state [12], trio coherent state [27] and in the interaction of intense laser beam with an inversion symmetric third order nonlinear medium [28]. From the fact that in first 15 years after its introduction, HOA was reported only in some particular cases, HOA appeared to be a very rare phenomenon. But recently we have shown that the HOA is not a rare phenomenon 29] and it can be seen in simple optical processes like six wave mixing process, four wave mixing process and second harmonic generation. To establish that further, here we have shown the existence of HOA in different intermediate states, namely, binomial state, reciprocal binomial state, photon added coherent state, hypergeometric state, Roy-Roy generalized binomial state and negative binomial state.

The present work is motivated by the recent experimental observation of intermediate state [14], theoretical observation of possibility of observing HOA in some simple optical systems [29 and the fact that the intermediate states, which frequently show different kind of nonclassicality, form a big family of quantum state. But till now HOA has been predicted only in one member (Shadowed negative binomial state) of such a big family of quantum states 12. Motivated by these facts the present work aims to study the possibility of HOA in all the popularly known intermediate states. The theoretical predictions of the present study can be experimentally verified with the help of various intermediate state generation schemes and homodyne experiment, since the criteria for HOA appears in terms of factorial moment, which can be measured by using homodyne photon counting experiments [30-33]. In the next section we have briefly described the criteria of HOA. In section 3 we have shown that the HOA of any arbitrary order can be seen in BS. In section 4 , Roy-Roy generalized binomial state [4] is studied and existence of HOA is predicted. Calculational details and methodology have been shown only in algebraically simple cases which are described in section 3 and 4 . Section 5 is divided in several subsections and we have followed the same procedure and have studied the possibilities of observing HOA in different intermediate states namely, reciprocal binomial state, negative binomial state, hypergeometric states and photon added coherent state. One subsection is dedicated for the discussion of one particular intermediate state. Finally section 6 is dedicated to conclusions.

\section{Criteria of HOA}

The criterion of HOA is expressed in terms of higher order factorial moments of number operator. There exist several criterion for the same which are essentially equivalent. Initially, using the negativity of $\mathrm{P}$ function [18], Lee introduced the criterion for HOA as

$$
R(l, m)=\frac{\left\langle N_{x}^{(l+1)}\right\rangle\left\langle N_{x}^{(m-1)}\right\rangle}{\left\langle N_{x}^{(l)}\right\rangle\left\langle N_{x}^{(m)}\right\rangle}-1<0,
$$


where $N$ is the usual number operator, $\left\langle N^{(i)}\right\rangle=\langle N(N-1) \ldots(N-i+1)\rangle$ is the ith factorial moment of number operator, \langle\rangle denotes the quantum average, $l$ and $m$ are integers satisfying the conditions $1 \leq m \leq l$ and the subscript $x$ denotes a particular mode. Ba An 27 choose $m=1$ and reduced the criterion of $l$ th order antibunching to

$$
A_{x, l}=\frac{\left\langle N_{x}^{(l+1)}\right\rangle}{\left\langle N_{x}^{(l)}\right\rangle\left\langle N_{x}\right\rangle}-1<0
$$

or,

$$
\left\langle N_{x}^{(l+1)}\right\rangle<\left\langle N_{x}^{(l)}\right\rangle\left\langle N_{x}\right\rangle
$$

Physically, a state which is antibunched in $l$ th order has to be antibunched in $(l-1) t h$ order. Therefore, we can further simplify (5) as

$$
\left\langle N_{x}^{(l+1)}\right\rangle<\left\langle N_{x}^{(l)}\right\rangle\left\langle N_{x}\right\rangle<\left\langle N_{x}^{(l-1)}\right\rangle\left\langle N_{x}\right\rangle^{2}<\left\langle N_{x}^{(l-2)}\right\rangle\left\langle N_{x}\right\rangle^{3}<\ldots<\left\langle N_{x}\right\rangle^{l+1}
$$

and obtain the condition for $l-t h$ order antibunching as

$$
d(l)=\left\langle N_{x}^{(l+1)}\right\rangle-\left\langle N_{x}\right\rangle^{l+1}<0 .
$$

This simplified criterion (7) coincides exactly with the physical criterion of HOA introduced by Pathak and Garica 28 and the criterion of Erenso, Vyas and Singh [34, recently Vogel has reported a class of nonclassicality conditions based on higher order factorial moments [35. All these criteria essentially lead to same kind of nonclassicality which belong to the class of strong nonclassicality according to the classification scheme of Arvind et al [36. Here we can note that $d(l)=0$ and $d(l)>0$ corresponds to higher order coherence and higher order bunching (many photon bunching) respectively. Actually, $\left\langle a^{\dagger l} a^{l}\right\rangle=\left\langle N^{(l)}\right\rangle$ is a measure of the probability of observing $l$ photons of the same mode at a particular point in space time coordinate. Therefore the physical meaning of inequalities (6) is that the probability of detection of single photon pulse is greater than that of a two photon in a bunch and that is greater than the probability of detection of three photon in a bunch and so on. This is exactly the characteristic that is required in a probabilistic single photon source used in quantum cryptography. In other words all the probabilistic single photon sources used in quantum cryptography should satisfy the criteria (7) of HOA [37.

\section{Binomial State}

Binomial state is originally defined as (1), from which it is straight forward to see that

$$
\begin{aligned}
a|p, M\rangle & =\sum_{n=0}^{M}\left\{\frac{M !}{(n-1) !(M-n) !} p^{n}(1-p)^{M-n}\right\}^{\frac{1}{2}} \mid n-1> \\
& \left.=\sum_{l=0}^{M-1}\left\{\frac{M(M-1) !}{l !(M-1-l) !} p^{l+1}(1-p)^{M-1-l}\right\}^{\frac{1}{2}} \mid l>\quad \text { (assuming } n-1=l\right) \\
& =[M p]^{\frac{1}{2}} \sum_{l=0}^{M-1}\left\{\frac{(M-1) !}{l !(M-1-l) !} p^{l}(1-p)^{M-1-l}\right\}^{\frac{1}{2}} \mid l> \\
& =[M p]^{\frac{1}{2}}|p, M-1\rangle .
\end{aligned}
$$

Similarly, we can write,

$$
\begin{aligned}
a^{2}|p, M\rangle & =\left[M(M-1) p^{2}\right]^{\frac{1}{2}}|p, M-2\rangle \\
a^{3}|p, M\rangle & =\left[M(M-1)(M-2) p^{3}\right]^{\frac{1}{2}}|p, M-3\rangle \\
\vdots & \vdots \\
a^{l}|p, M\rangle= & {\left[M(M-1) \ldots(M-l+1) p^{l}\right]^{\frac{1}{2}}|p, M-l\rangle } \\
& =\left[\frac{M !}{(M-l) !} p^{l}\right]^{\frac{1}{2}}|M-l, p\rangle .
\end{aligned}
$$

Therefore,

$$
\langle M, p| a^{\dagger l}=\langle M-l, p|\left[\frac{M !}{(M-l) !} p^{l}\right]^{\frac{1}{2}}
$$


and consequently,

$$
\left\langle M, p\left|n^{(l)}\right| p, M\right\rangle=\left\langle M, p\left|a^{\dagger l} a^{l}\right| p, M\right\rangle=\left[\frac{M !}{(M-l) !} p^{l}\right] .
$$

Now substituting (11) in equation (7) we obtain the condition for $l$ th order antibunching as

$$
d(l)=\left[\frac{M !}{(M-l-1) !} p^{l+1}\right]-[M p]^{l+1}<0
$$

or,

$$
(M-1)(M-2) \ldots .(M-l)<M^{l}
$$

which is always satisfied for any $M>l$ and both $M$ and $l$ are positive (since every term in left is $<M$ ). As $M$ is the number of photons present in the field and $d(l)$ is a measure of correlation among $(l+1)$ photons, therefore $M \geq(l+1)$ or $M>l$. Consequently, a binomial state always shows HOA and the highest possible order of antibunching that can be seen in a binomial state is equal to $M-1$, where $M$ is the number of photon present in the field. From $(12)$ it is straight forward to see that the number state is always higher order antibunched and in the other extreme limit (when $p \rightarrow 1, M \rightarrow \infty$ and the BS reduces to coherent state) $d(l)=0$, which is consistent with the physical expectation.

\section{Generalized Binomial State}

We have already mentioned that there are different form of generalized binomial states 3.5 . For the present study we have chosen generalized binomial state introduced by Roy and Roy 4 . They have introduced the generalized binomial state (GBS) as

$$
|N, \alpha, \beta\rangle=\sum_{n=o}^{N} \sqrt{\omega(n, N, \alpha, \beta)}|n\rangle
$$

where,

$$
\omega(n, N, \alpha, \beta)=\frac{N !}{(\alpha+\beta+2)_{N}} \frac{(\alpha+1)_{n}(\beta+1)_{N-n}}{n !(N-n) !}
$$

with $\alpha, \beta>-1, n=0,1, \ldots, N$, and

$$
(a)_{0}=1 \quad(a)_{n}=a(a+1) \ldots(a+n-1) .
$$

This intermediate state reduces to vacuum state, number state, coherent state, binomial state and negative binomial state in different limits of $\alpha, \beta$ and $N$. In order to obtain an analytic expression of $d(l)$ for this particular generalized binomial state we need to prove following useful identity:

Identity1:

$$
a(a+1)_{n}=(a)_{n+1}
$$

Proof: Using 16 we can write

$$
(a+1)_{n}=(a+1) \ldots(a+n)=\frac{a(a+1) \ldots(a+n)}{a}=\frac{(a)_{n+1}}{a} .
$$

Therefore,

$$
a(a+1)_{n}=(a)_{n+1} .
$$

Now it is easy to see that the above identity 17 yields the following useful relations:

$$
(\alpha+1)_{l+1}=(\alpha+1)(\alpha+2)_{l}
$$

and

$$
(\alpha+\beta+2)_{N}=(\alpha+\beta+2)(\alpha+\beta+3)_{N-1}=(\alpha+\beta+2)(\alpha+2+\beta+1)_{N-1} .
$$


Using (14) and 15 we can obtain

$$
\begin{aligned}
a|N, \alpha, \beta\rangle & =\sum_{n=0}^{N}\left\{\frac{N !}{(\alpha+\beta+2)_{N}} \frac{(\alpha+1)_{n}}{(n-1) !} \frac{(\beta+1)_{N-n}}{(N-n) !}\right\}^{\frac{1}{2}}|n-1\rangle \\
& =\sum_{l=0}^{N-1}\left\{\frac{N(N-1) !}{(\alpha+\beta+2) N} \frac{(\alpha+1)_{l+1}}{l !} \frac{(\beta+1)_{N-1-l}}{(N-1-l) !}\right\}^{\frac{1}{2}}|l\rangle
\end{aligned}
$$

where $n=l-1$ has been used. Now we can apply (18) and $(19)$ on 20 to obtain

$$
\begin{aligned}
a|N, \alpha, \beta\rangle & =\left\{\frac{N(\alpha+1)}{(\alpha+\beta+2)}\right\}^{\frac{1}{2}} \sum_{l=0}^{N-1}\left\{\frac{(N-1) !(\alpha+2)_{l}(\beta+1)_{N-1-l}}{(\alpha+2+\beta+1)_{N-1} l !(N-1-l) !}\right\}^{\frac{1}{2}}|l\rangle \\
& =\left\{\frac{N(\alpha+1)}{(\alpha+\beta+2)}\right\}^{\frac{1}{2}} \sum_{n=0}^{N-1} \sqrt{\omega(n, N-1, \alpha+1, \beta)}|n\rangle,
\end{aligned}
$$

where dummy variable $l$ is replaced by $n$. Therefore,

$$
\begin{aligned}
\left\langle N, \alpha, \beta\left|a^{\dagger} a\right| N, \alpha, \beta\right\rangle & =\frac{N(\alpha+1)}{(\alpha+\beta+2)} \\
\left\langle N, \alpha, \beta\left|a^{\dagger 2} a^{2}\right| N, \alpha, \beta\right\rangle & =\frac{N(N-1)(\alpha+1)(\alpha+2)}{(\alpha+\beta+2)(\alpha+\beta+3)} \\
\vdots & \vdots \\
\left\langle N, \alpha, \beta\left|a^{\dagger l} a^{l}\right| N, \alpha, \beta\right\rangle & =\frac{[N(N-1) \ldots \ldots(N-l+1)][(\alpha+1)(\alpha+2) \ldots . .(\alpha+l)]}{(\alpha+\beta+2)(\alpha+\beta+3) \ldots \ldots(\alpha+\beta+l+1)} \\
& =\frac{N !(\alpha+l) !(\alpha+\beta+1) !}{(N-l) ! \alpha !(\alpha+\beta+l+1) !}
\end{aligned}
$$

and

$$
\begin{aligned}
d_{G B S}(l) & =\frac{N !(\alpha+l+1) !(\alpha+\beta+1) !}{(N-l-1) ! \alpha !(\alpha+\beta+l+2) !}-\left\{\frac{N(\alpha+1)}{(\alpha+\beta+2)}\right\}^{l+1} \\
& =\frac{[N(N-1) \ldots \ldots(N-l)][(\alpha+1)(\alpha+2) \ldots \ldots(\alpha+l+1)]}{(\alpha+\beta+2)(\alpha+\beta+3) \ldots . .(\alpha+\beta+l+2)}-\left\{\frac{N(\alpha+1)}{(\alpha+\beta+2)}\right\}^{l+1}
\end{aligned}
$$

The physical condition $N \geq l+1$ ensures that all the terms in $d(l)$ are positive. The expression of $d(l)$ is quite complex and it depends on various parameters (e.g. $\alpha, \beta$ and $N$ ). Fig 1 shows that for particular values of these parameters HOA is possible. As it is expected from the earlier works on the properties of HOA [28], the depth of nonclassicality is more in case of $d_{G B S}(9)$ than in $d_{G B S}(8)$. This is consistent with earlier observation. A systematic study reveals that the probability of observing HOA increases with the increase of $\alpha$ but it decreases (i.e the probability of higher order bunching increases) with the increase of $\beta$. This can be seen clearly in Fig2 and Fig3. Further it is observed (from Fig1 and Fig2) that for lower values of $\alpha$ the probability of bunching increases with the increase of $N$ but for a comparatively large values of $\alpha$ (larger compare to $\beta$ ) the probability of HOA increases with the increase in $N$ [see Fig 2] but the situation is just opposite in the case of $\beta$ (as it is seen from Fig3).

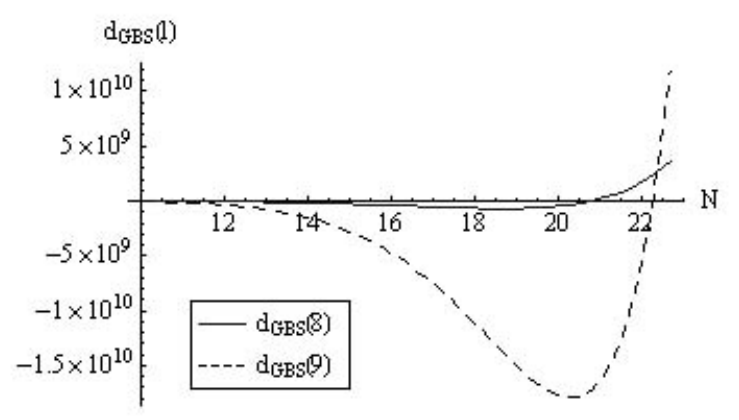

Figure 1: Higher order antibunching can be seen in Generalized binomial state. Existence of 8th and 9th order antibunching (for $\alpha=2$ and $\beta=1$ )and variation of depth of nonclassicality with $N$ has been shown.

While studying different limiting cases of Roy and Roy generalized binomial state, we have observed that binomial state and number state always show HOA and $d(l)=0$ for coherent state. This is consistent with the physical expectation and the conclusions of the last section. 


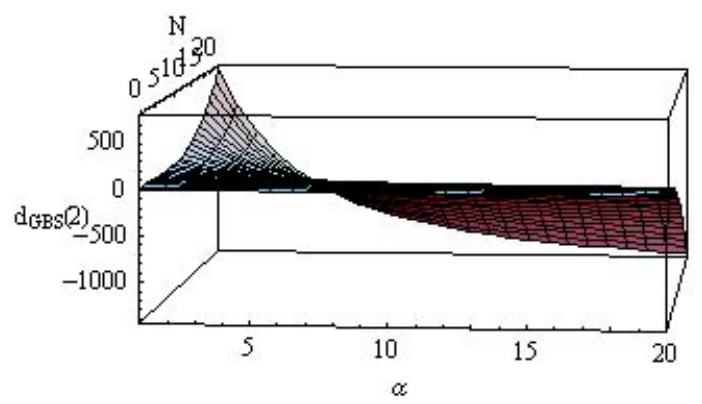

Figure 2: Variation of $d_{G B S}(2)$ with $\alpha$ and $N$ for $\beta=1$.

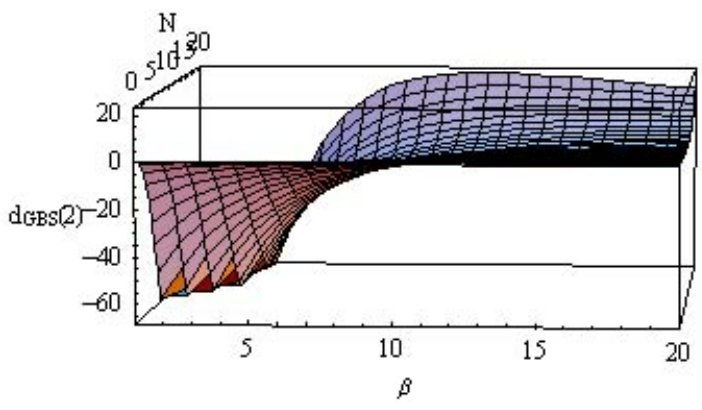

Figure 3: Variation of $d_{G B S}(2)$ with $\beta$ and $N$ for $\alpha=10$.

\section{$5 \quad$ Other Intermediate States}

As it is mentioned in the earlier sections, there exist several different intermediate states. For the systematic study of possibility of observing HOA in intermediate states, we have studied all the well known intermediate states. Since the procedure followed for the study of different states are similar, mathematical detail has not been shown in the subsections below. But from the expression of $d(l)$ and the corresponding plots it would be easy to see that the HOA can be observed in all the intermediate states studied below.

\subsection{Reciprocal binomial state}

Reciprocal binomial state (RBS) can be defined as 10

$$
|\phi\rangle=\frac{1}{N} \sum_{k=0}^{N}\left(\begin{array}{c}
N \\
K
\end{array}\right)^{-1 / 2} e^{i k(\theta-\pi / 2)}|k\rangle
$$

where $N$ is a normalization constant. Using antinormal ordering and procedure adapted in section 3 , we can obtain;

$$
\begin{aligned}
d_{R B S}(l) & =\sum_{i=0}^{l+1}(-1)^{i} \frac{(l+1) !^{2}}{(l+1-i) !^{2} i !} \frac{(N+(l+1-i) !}{N !}-N^{l+1} \\
& =\frac{\pi \csc (\pi(l+N))(l+1) !^{2} \operatorname{Gamma}(l+1-N)}{M !(\operatorname{Gamma}(2+l) \operatorname{Gamma}(-N))^{2}}-N^{l+1}
\end{aligned}
$$

Where Gamma denotes the Gamma function. The possibility of observing HOA in reciprocal binomial state can be clearly seen from the Figure 4 . But it is interesting to note that the nature of singularity and zeroes present in the simplified expression of $d_{R B S}(l)$ as expressed in the last line of (24) can provide us some important information. For example, the underlying mathematical structure of the criterion of HOA and that of reciprocal binomial state demands that $l$ and $N$ be integers but if both of them are integer then $d_{R B S}(l)$ has a singularity as the $\csc [\pi(l+N)]$ term present in the numerator blows up. But this local 
singularity can be circumvented by assuming $l \rightarrow$ integer and $N \rightarrow$ integer. In this situation (i.e. when $l$ and $N$ tends to integer value) $\left\langle N^{(l)}\right\rangle$ is finite and consequently $d(l)$ is also finite. This is the reason that the singular nature of the simplified expression of $d_{R B S}(l)$ is not reflected in the Figure 4 . In the analysis of the $d_{R B S}(l)$ it is also interesting to observe that $\operatorname{Gamma}(-N)=\infty$ for $N=$ integer, and in an approximated situation when $l \rightarrow$ integer and $M=$ integer, the $\csc [\pi(l+N)]$ term in the numerator is no more singular and as a result $\left\langle N^{(l)}\right\rangle=0$ and $d_{R B S}=-N^{(l+1)}$. In this situation one can observe HOA for arbitrarily large values of $l$ and $N$. Thus physically, it is expected that in reciprocal binomial state higher order antibunching of any arbitrary order will be seen and HOA will not be destroyed with the increase of $N$, as it happens (for some particular values of $\alpha$ and $\beta$ ) in the case of generalized binomial state (see Figure 1).

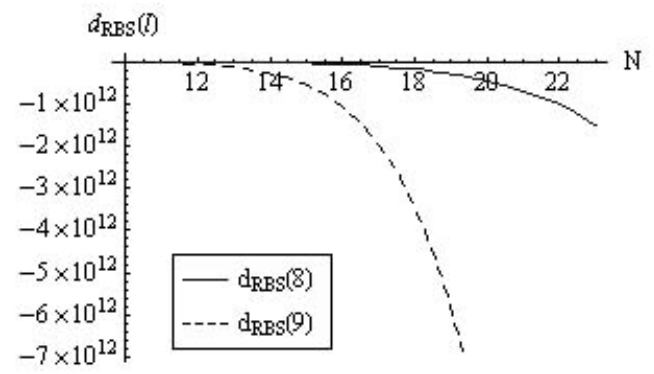

Figure 4: Variation of $d_{R B S}(8)$ and $d_{R B S}(9)$ with photon number $N$

\subsection{Negative Binomial State}

Following Barnett $[38$ we can define Negative Binomial state (NBS) as

$$
|\eta, M\rangle=\sum_{n=M}^{\infty} C_{n}(\eta, M)|n\rangle
$$

where $C_{n}(\eta, M)=\left[\left(\begin{array}{c}n \\ M\end{array}\right) \eta^{M+1}(1-\eta)^{n-M}\right]^{1 / 2}, 0 \leq \eta \leq 1$ and $\mathrm{M}$ is a non-negative integer. This intermediate state interpolates between number state and geometric state. Following the mathematical techniques adopted in the earlier sections we obtain

$$
d_{N B S}(l)=\eta^{-l}\left(\frac{(l+M+1) !{ }_{2} F_{1}(-l-1,-l-1 ;-l-M-1 ; \eta)}{M !}-\frac{(M+1)^{l+1}}{\eta}\right)
$$

where ${ }_{2} F_{1}(a, b ; c ; z)$ is a conventional hypergeometric function. Variation of $d(l)$ with various parameters such as $\eta, l$ and $M$ have been studied and are shown in Figure 5- Figure6. From these figures one can observe that the state is not always antibunched, rather the plot of $d_{N B S}(8)$ has a very sharp rise near $\eta \approx .15$ and $M \approx 10$. From Figure 5 we can observe that the possibility of higher order bunching get destroyed with the increase of $M$ or $\eta$. The second feature has clearly been shown in Figure 6 , where we can see that $d_{N B S}(8)$ become positive for a small region for $M=10$ but for a bigger region $(\eta \approx .3$ to $\eta=1)$ it remains negative and thus shows the existence of HOA. The broad features remain same for the other orders (other values of l) of antibunching.

In the limit $N \rightarrow 0$ the negative binomial state reduces to geometric state (GS). In this limit $d_{N B S}(l)$ reduces to

$$
d_{G S}(l)=\frac{1}{\eta^{l+1}}\left((1-\eta)^{l+1} \eta(l+1) !-1\right) .
$$

It is interesting to observe that the above expression has a singularity at $\eta=0$ and $d_{G S}(l) \rightarrow-\infty$ in the limit $\eta \rightarrow 0$. Consequently one negative values of $d_{G S}$ can be seen at very very small values $\eta$ but this is not the signatures of HOA, rather this is the signature of the existence of a strong singularity in the neighborhood. This can further justified by the fact that for any finite value of $l$ there does not exist any real root, (whose 


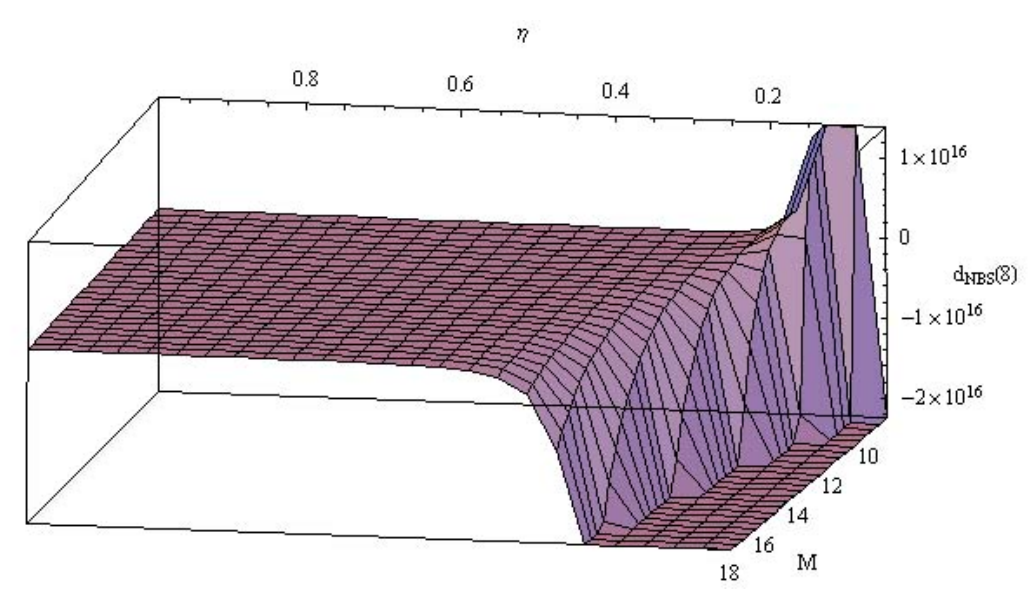

Figure 5: Variation of $d_{N B S}(8)$ with $\eta$ and $M$
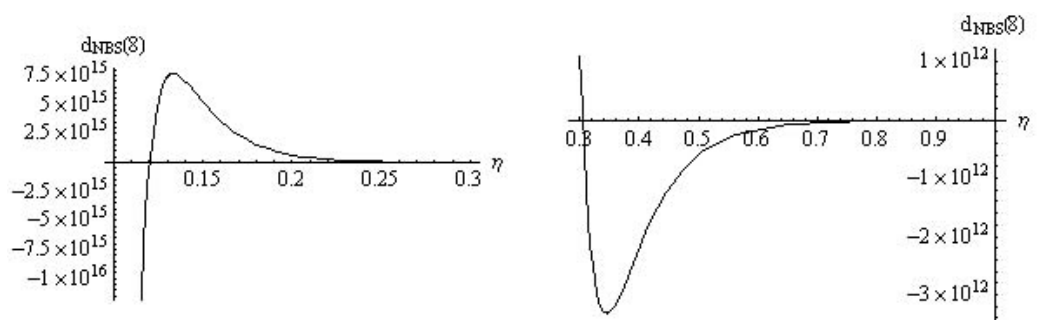

Figure 6: Variation of $d_{N B S}(8)$ with $\eta$ for $M=10$

value is close to zero or which is negligibly small compared to 1$)$ of $d_{G S}(l)=0$. Thus there is no oscillation between bunching and antibunching. We further observe that $d_{G S}(l) \rightarrow-1$ in the limit $\eta \rightarrow 1$ and for $l \geq 3$ there exists only one physically acceptable real root of $d_{G S}(l)=0$. By physically acceptable real root we mean that it lies in $[0,1]$. Before this value of $\eta$ (or before the physically acceptable real root) the state shows higher order super poissonian photon statistics but immediately after the root it becomes negative and thus shows HOA or higher order subpoissonian photon statistics. As we increase $l$ the real root shifts in right side of the real axis (i.e towards $\eta=1$ ). It can be clearly seen in the Figure 7 . From this figure it can be easily seen that it satisfies all the physical properties of HOA derived in 28 .

\subsection{Photon added coherent state}

Photon added coherent state (2) or PACS, which was introduced by Agarwal and Tara 13 can be defined as

$$
|\alpha, m\rangle=\frac{\exp \left(-\frac{|\alpha|^{2}}{2}\right)}{\sqrt{L_{m}\left(-|\alpha|^{2}\right) m !}} \sum_{n=0}^{\infty} \alpha^{n} \frac{\sqrt{(m+n) !}}{n !}|n+m\rangle
$$

where $L_{m}(x)$ is Lauguere polynomial of $m t h$ order. Rigorous operator algebra yields

$$
\begin{aligned}
d_{P A C S}(l) & =\frac{\exp \left(-\alpha^{2}\right) \alpha^{2 l+2}((l+m+1) !)^{2}{ }_{P} F_{Q}\left(\{1,2+l+m, 2+l+m\} ;\{2+l, 2+l, m+1\} ; \alpha^{2}\right)}{(m !(l+1) !)^{2}{ }_{1} F_{1}\left(-m ; 1 ;-\alpha^{2}\right)} \\
& -\left(\frac{\exp \left(-\alpha^{2}\right)\left(-m+m_{1} F_{1}\left(1+m ; 1 ; \alpha^{2}\right)+(1+m) \alpha^{2}{ }_{1} F_{1}\left(2+m ; 2 ; \alpha^{2}\right)\right)}{1 F_{1}\left(-m ; 1 ;-\alpha^{2}\right)}\right)^{l+1},
\end{aligned}
$$

where, ${ }_{P} F_{Q}$ is the generalized Hypergeometric function. The analytic expression for $d_{P A C S}(l)$ is quite complicated and it is difficult to conclude anything regarding its photon statistics directly from (29) but we have investigated the variation of $d_{P A C S}$ with $\alpha, l$ and $m$ and could not find any region which does not show HOA. Therefore, HOA can be seen in this particular intermediate state. This fact is manifested in Fig 8 and Fig 9. From these figures it is easy to observe that depth of nonclassicality increases monotonically 


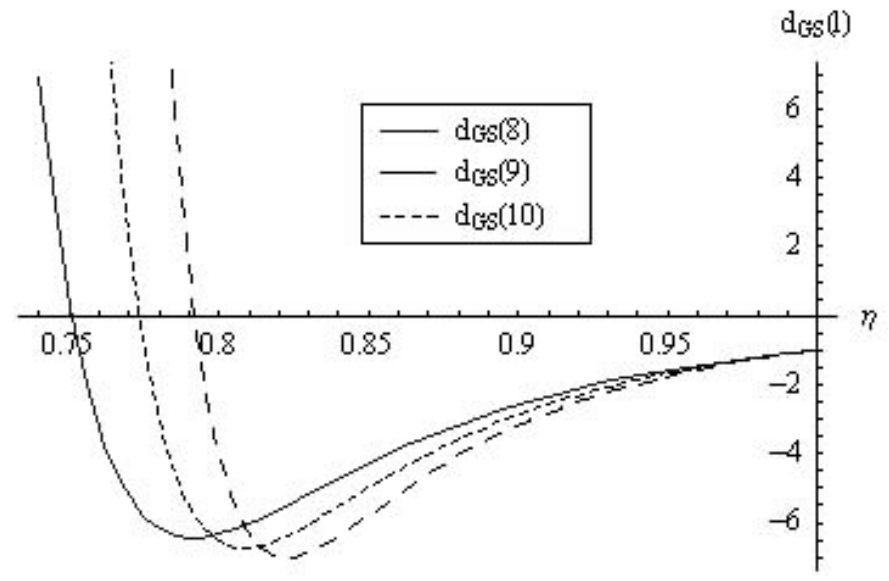

Figure 7: Variation of $d_{G S}(8), d_{G S}(9)$ and $d_{G S}(10)$ with respect to $\eta$.

with the increase of $m$ and $l$. The variation of depth of nonclassicality with $\alpha$ has a deep for a small value of $\alpha$ (see Fig 8. and Fig. 9). Although $d_{P A C S}$ is always negative, initially its magnitude $\left(d_{P A C S}(l)\right.$ without the negative sign) increases, then decreases and then become a monotonically increasing function. Actually for the smaller values of $\alpha$, an effective contribution from the combination of all the hypergeometric functions appears and dominates but as soon as $\alpha$ increases a bit, the $\exp \left(-\alpha^{2}\right)$ term starts dominating and as a consequence depth of nonclassicality increases monotonically. Here we would also like to note that in contrast to the photon added coherent state, $d(l)$ is always positive for the analogous state $|\alpha,-m\rangle$, introduced by Sivakumar [39. Thus $|\alpha,-m\rangle$ always shows higher order superpoisonian photon statistics. Further, we would like to note that photon added coherent state which, is intermediate between coherent and fock state has already been experimentally generated in 2004 [14]. Therefore, it is technically feasible to observe higher order antibunching for an intermediate state.

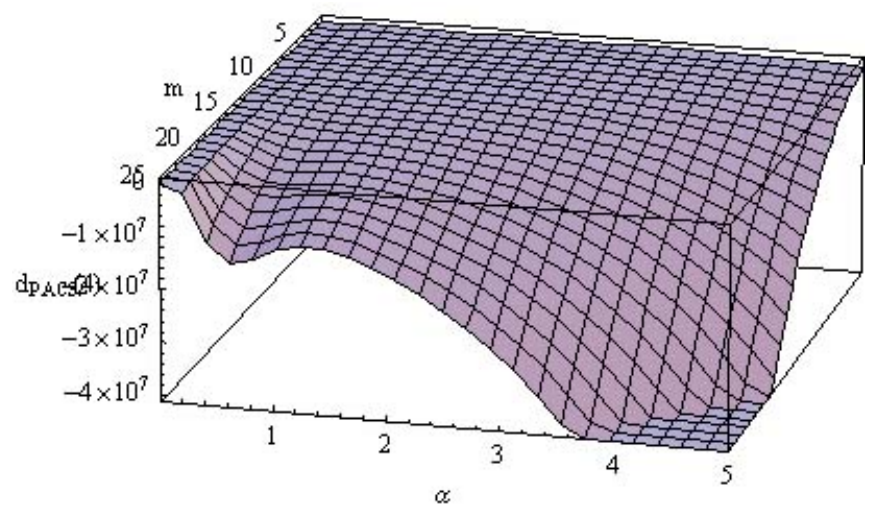

Figure 8: Variation of $d_{P A C S}(4)$ with $\alpha$ and $m$

\subsection{Hypergeometric state}

Following 8 hypergeometric state (HS) can be defined as

$$
|L, M, \eta\rangle=\sum_{n=0}^{M} H_{n}^{M}(\eta, L)|n\rangle
$$




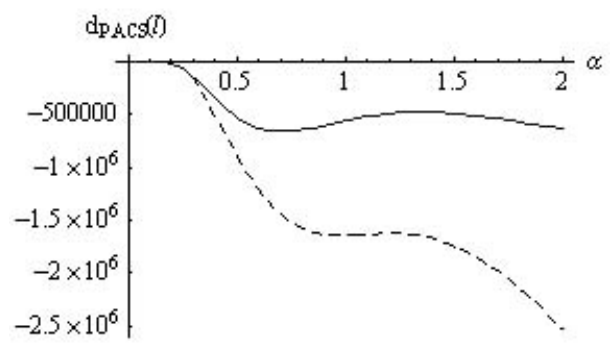

Figure 9: Variation of $10 d_{P A C S}(3)$ and $d_{P A C S}(4)$ with $\alpha$ for $m=15$, the solid line denotes $d_{P A C S}(3)$ and the dashed line denotes $d_{P A C S}(4)$ to keep show the variation in the same scale $d_{P A C S}(3)$ is multiplied by 10. The plot shows that depth of nonclassicality of $d_{P A C S}(4)$ is always greater than that of $d_{P A C S}(3)$ which is consistent with the properties of HOA

where the probability $\eta$ is a real parameter satisfying $0<\eta<1 . L$ is a real number satisfying

$$
\begin{gathered}
L \geq \max \left\{M \eta^{-1}, M(1-\eta)^{-1}\right\}, \\
H_{n}^{M}(\eta, L)=\left[\left(\begin{array}{c}
L \eta \\
n
\end{array}\right)\left(\begin{array}{c}
L(1-\eta) \\
M-n
\end{array}\right)\right]^{\frac{1}{2}}\left(\begin{array}{c}
L \\
M
\end{array}\right)^{-\frac{1}{2}},
\end{gathered}
$$

and

$$
\left(\begin{array}{c}
\alpha \\
n
\end{array}\right)=\frac{\alpha(\alpha-1) \ldots(\alpha-n+1)}{n !}, \quad\left(\begin{array}{c}
\alpha \\
0
\end{array}\right) \equiv 1 .
$$

Here $\alpha$ is not necessarily an integer. Using the techniques adopted in the earlier sections and a bit of operator algebra we can obtain a closed form analytic expression for $d(l)$ as

$$
d_{H S}(l)=-(M \eta)^{l+1}+\frac{(L-l-1) ! M !(L \eta) !}{L !(M-l-1) !(L \eta-1-l) !}
$$

From Fig. 10 it is clear that HOA can be observed in hypergeometric state. It is also observed that the depth of nonclassicality increases with the increase in $\eta$ and $M$. Hypergeometric state reduces to binomial state, coherent state, number state and vacuum state in different limits of $M, L$ and $\eta$. It has been verified that if we impose those limits on $d_{H S}$ then we obtain corresponding photon statistics.

\section{Conclusions}

In essence all the intermediate states studied in the present paper show HOA i.e. higher order subpoissonian photon statistics. But it neither mean that all the intermediate states are higher order antibunched (for example $|\alpha,-m\rangle$ is always higher order bunched) nor an intermediate state which shows HOA will show it for all possible values of the control parameters (for example negative binomial state and generalized binomial state shows both higher order bunching and higher order antibunching for various parametric values). Thus we can conclude that, as far as HOA is concerned there does not exist any common characteristics among the different intermediate states but most of them show HOA. Further, we have seen from (Figure1-Figure10) that the depth of nonclassicality of a higher order antibunched state varies with different control parameters (e.g. $\alpha, N, m$ etc.). These parameters represent some physical quantity and their value may be controlled and consequently by controlling these parameters we can control the depth of nonclassicality. Photon statistics (factorial moment) of an intermediate state can be obtained experimentally by using homodyne detection (photon counting) technique. These facts along with the recent success in experimental production of intermediate state open up the possibility of experimental observation of HOA in intermediate state. Thus the present work strongly establishes the fact that HOA is not a rare phenomenon.

The prescription followed in the present work is easy and straight forward and it can be used to study the possibilities of observing higher order antibunching in other intermediate states (such as negative hyper 


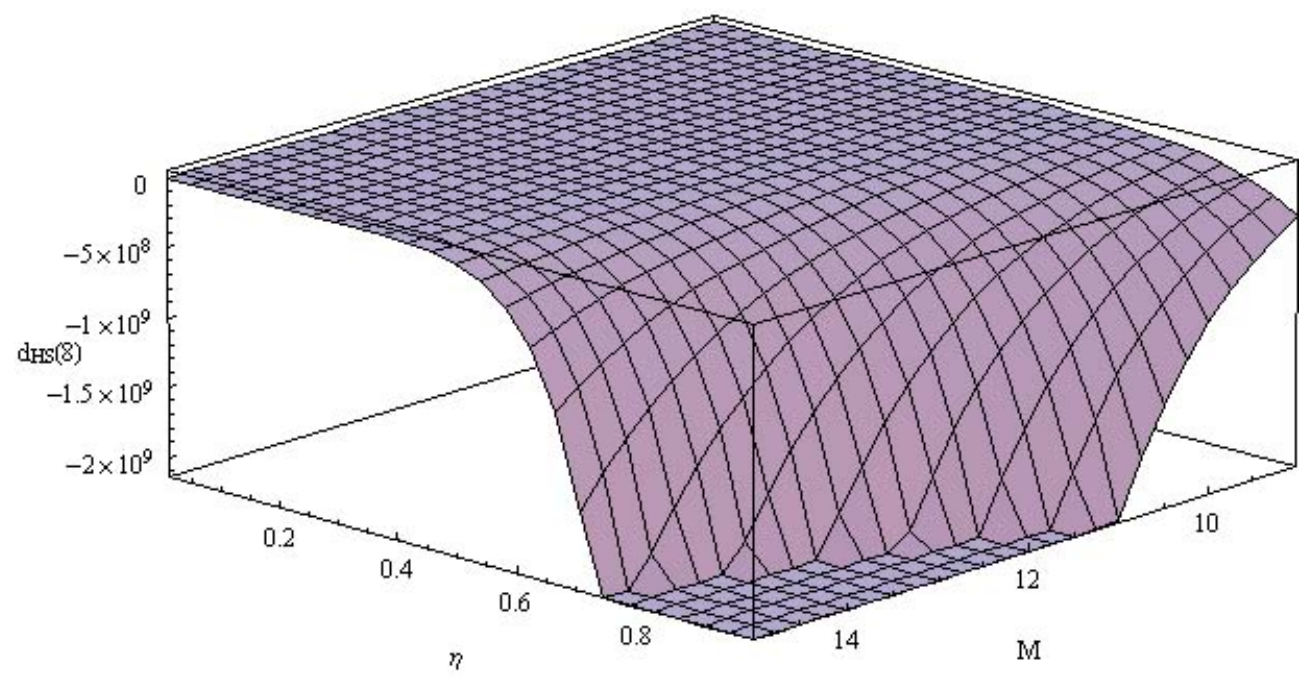

Figure 10: Variation of $d_{H S}(8)$ with $\eta$ and $M$, when lowest allowed values of $L$ have been chosen at every point.

geometric state, excited binomial state and odd excited binomial state) and other physical systems. Thus it opens up the possibility of studying higher order nonclassical effects from a new perspective. This is also important from the application point of view because any probabilistic single photon source used for quantum cryptography has to satisfy the condition for higher order antibunching.

Acknowledgement: AP thanks to DST, India for partial financial support through the project grant $\mathrm{SR} \backslash \mathrm{FTP} \backslash \mathrm{PS}-13 \backslash 2004$.

\section{References}

[1] D Stoler, B E A Saleh and M C Teich, Opt. Acta 32 (1985) 345.

[2] A Vidiella and J A Roversi, Phys. Rev. A 50 (1994) 5233.

[3] Hong-Chen Fu and Ryu Sasaki J. Phys. A 29 (1996) 5637.

[4] P Roy and B Roy, J. Phys. A 30 (1997) L719.

[5] Hong-Yi Fan and Nai-le Liu, Phys. Lett. A 264 (1999) 154.

[6] X G Wang and H C Fu, Int. J. Theo. Phys. 39 (2000) 1437.

[7] A S F Obada, M Darwish and H H Salah, Int. J. Theo. Phys. 41 (2002) 1755.

[8] H C Fu and R Sasaki, J. Math. Phys. 38 (1997) 2154.

[9] H Fan and N Liu, Phys. Lett. A 250 (1998) 88.

[10] M H Y Moussa and B Baseia, Phys. Lett. A 238 (1998) 223.

[11] C T Lee, Phys. Lett A, 229 (1997) 413.

[12] R Srinivasan and C T Lee, Phys. Lett A, 218 (1996) 151.

[13] G S Agarwal and K Tara, Phys. Rev. A, 43 (1991) 492.

[14] A Zavatta, S Viciani, M Bellini, Science 306 (2004) 660. 
[15] C Valverde et al, Phys. Lett. A, 315 (2003) 213.

[16] R Lo Franco et al, Phys. Rev. A 74 (2006) 045803.

[17] R Lo Franco et al, Open Sys. \& Information Dyn. 13 (2006) 463.

[18] Meystre P and Sargent M III, Elements of Quantum Optics second edition Springer-Verlag, Berlin 1991.

[19] Dodonov V V J. Opt. B. Quant. and Semiclass. Opt. 4 (2002) R1.

[20] Hanbury-Brown R, Twiss R Q, Nature 177 (1956) 27.

[21] Hong C K and Mandel L, Phys. Rev. Lett. 54 (1985) 323.

[22] Hong C K and Mandel L, Phys. Rev. A 32 (1985) 974.

[23] Lee C T, Phys. Rev. A 411721 (1990).

[24] Lee C T, Phys. Rev. A 411569 (1990).

[25] M. Hillery, Phys. Rev. A 363796 (1987).

[26] D K Giri and P S Gupta, Opt. Comm. 221 (2003) 135.

[27] An N B, J. Opt. B: Quantum Semiclass. Opt. 4 (2002) 222.

[28] Pathak A and Garcia M, Applied Physics B 84 (2006) 484.

[29] P.Gupta, P. Pandey and A. Pathak, J. Phys. B 39 (2006) 1137, (quant-ph/0508060).

[30] Bachor H A, A Guide to Experiments in Quantum Optics, Wiley-vch, Weinheim 1998, Chapter 8 and Chapter 10.

[31] W Vogel, D Welsch, S Wallentowitz, Quantum Optics an Introduction, Second edition, Wiley-vch, Berlin 2001, Chapter 6.

[32] D Erenso, R Vyas and S Singh, J. Opt. Soc. B 19 (2002) 1471.

[33] R Vyas and S Singh, Phys. Rev. A 40 (1989) 5147.

[34] Erenso D, Vyas R and Singh S, J. Opt. Soc. Am. B 19 (2002) 1471

[35] EV Shchukin, W Vogel - Physical Review A, 72 (2005) 043808.

[36] Arvind, N Mukunda and R Simon, Phys. Rev A, 56 (1997) 5042.

[37] A Pathak, Ind. J. Phys. 80 (2006) 495.

[38] S M Barnett, J. Mod. Opt A 45 (1998) 2201. (1999) 3441.

[39] S Shivakumar, J. Phys. A 32 (1999) 3441. 